\title{
The Phytochemical Content and the In vitro Antifungal Properties of Senna alata (Linn.) Roxb.: A Review
}

\author{
Charisse Leanne B. Legaspi ${ }^{1}$ and Cecilia C. Maramba-Lazarte ${ }^{1,2}$ \\ ${ }^{1}$ Institute of Herbal Medicine, National Institutes of Health, University of the Philippines Manila \\ ${ }^{2}$ Department of Pharmacology and Toxicology, College of Medicine, University of the Philippines Manila
}

\begin{abstract}
Infections caused by invasive fungal species continue to rise due to various contributing factors including the changes in the environmental and weather conditions, lifestyle, the occurrence of natural disasters, and the weakened human immune system. Even though synthetic drugs effectively cure fungal diseases, their unwanted side effects, as well as the rapid rise in resistance, have compelled researchers to develop new antifungal agents. Several medicinal plants are folklorically known to have antifungal activities. Among the traditionally used antifungal herbal plants is Senna alata, commonly known as akapulko and ringworm bush. In the current review, phytochemical analysis and numerous non-clinical studies on akapulko have been performed and confirmed its activity against several fungi pathogenic to humans. Anthraquinone compounds seemed to be the major phytochemicals responsible for its antifungal activity. In the Philippines, clinical trials have also confirmed its utility as a topical agent in treating cutaneous fungal infections. Research gaps that need to be addressed include the determination of the exact molecular mechanisms of their fungal killing action.
\end{abstract}

Key Words: antifungal, Senna alata, akapulko

\section{INTRODUCTION}

Diseases and infections caused by invasive fungal species have continued to rise and emerge. Changes in the environment, different lifestyles, the shifting weather conditions, ability of the fungi to alter its virulence factors, compromised immune system of the host, seasonal migration, and occurrence of natural disasters were cited as the major contributing factors for fungal invasion., ${ }^{1,2}$ Majority of the reports for infections were due to newly discovered fungi and even re-emerging fungal pathogens that can reconstruct their virulence factors. ${ }^{1}$ In the Philippines, the warm and humid tropical climate, and the age, type of occupation, genetic susceptibility, and immune sensitivity of the Filipinos favor fungal infection pervasiveness. The most common fungal infection cases in the country include pityriasis versicolor $(25.34 \%)$, tinea corporis $(22.63 \%)$, tinea cruris $(16.7 \%)$, and tinea pedis $(16.38 \%)^{3}$; in $2017,1.9 \%$ of the population suffers from serious fungal infections. ${ }^{4}$

Countering the effect and spread of fungal infections, commercially available antifungal drugs such as the polyenes (amphotericin B) and azoles (fluconazole, itraconazole, voriconazole, and posaconazole) disrupt fungal cell wall stability and destroy the pathogen's homeostasis causing osmotic stress. ${ }^{5}$ Amphotericin $\mathrm{B}$ is the major choice

in treating fungal cystitis, peritonitis, dermatoses, and

Corresponding author: Charisse Leanne B. Legaspi, MSc Institute of Herbal Medicine

National Institutes of Health

University of the Philippines Manila

623 Pedro Gil St., Ermita, Manila 1000, Philippines

Email: cblegaspi1@up.edu.ph 
intraocular infections and is effective against Candida albicans ${ }^{5,6,7}$ by binding to the plasma membrane component ergosterol causing cell leakage and death. On the other hand, azoles, a group of broad-spectrum fungistatic agents, inhibit the enzymes for ergosterol synthesis, an important enzyme for fungal cell wall biosynthesis., ${ }^{5,8}$ While deemed effective, these synthetic drugs have features that limit their use. The nephrotoxicity caused by Amphotericin B ${ }^{9,10}$ is a well-known side effect; in the use of azoles, it requires cytochrome P450-induced oxidative metabolism to be eliminated from the body. ${ }^{8,11}$ Other reported side effects of azoles were nausea, vomiting, headache, abdominal pain, skin infections, occasional increase in liver serum enzymes, pruritus, hepatotoxicity, and gynecomastia. ${ }^{12}$

Considering that these synthesized drugs have its share of negative side effects and are expensive, and that fungi continually develop resistance against them, the search for alternative sources of therapy is necessary. Medicinal plants have long been used as a source of bioactive compounds for modern medications. Those with folkloric use as an antifungal aid were most likely candidates for the discovery of treatments for fungal infections. Among the plants traditionally used for fungal infection is Senna alata (Linn.) Roxb. (synonym Cassia alata Linn.) of the Family Fabaceae/Leguminosae), more commonly known as akapulko, ringworm bush, Christmas candle, candle brush, or calabra brush. ${ }^{13,14}$

Akapulko is an erect perennial shrub, originally cultivated as an ornament ${ }^{15,16}$ native to Southeast Asia (Japan and Indonesia), Africa (Ghana, Nigeria), Northern Australia, and Latin America (Mexico). ${ }^{17,18,19}$ It is traditionally used as a laxative, purgative, and as treatment to skin problems. ${ }^{14,16,20}$ The commonly used parts were the leaves, bark, stem, root, pod, and seeds ${ }^{21}$; however, majority of the documented reports used the leaves and the roots. The leaves were said to be sudorific, diuretic and purgative, ${ }^{22,23}$ and its decoction can treat bronchitis and asthma. ${ }^{23} \mathrm{On}$ the other hand, the roots were traditionally used as a pain reliever for dysmenorrhea, stomach pain during pregnancy, dysentery, convulsion, heart failure, edema, jaundice, and paralysis in tropical Africa. ${ }^{24}$

\section{Phytochemicals Responsible for its Antifungal Action}

Phytochemicals are biologically active compounds produced by plants, dedicated for their survival and adaptation. There are five general classifications of phytochemicals: carbohydrates, lipids, terpenoids, phenolic acids, and alkaloids and other nitrogen-containing metabolites. ${ }^{25}$ In most cases, the type and concentration of the phytochemicals present in a specific plant is a product of different factors such as the type of soil where it was planted, cultivation method, type of fertilizer used, the weather condition, season, and even with the time of the day. In addition, the amount of total flavonoids, phenolics, flavonols, condensed tannins, and carotenoids that can be extracted from a plant is affected by the type, concentration, $\mathrm{pH}$, polarity, and temperature of solvent used for extraction, plant part used, age of the plant, genotype or variety of the plant, and the type of extraction method performed (e.g. maceration, homogenization, etc.). ${ }^{26,27,28}$ A recent study showed that phytochemical concentration, specifically of the glucosinolates and the antioxidant activity of the two genotypes of cauliflower changed through-out the threeyear study. ${ }^{29}$ The scarce rainfall during the third year of their study showed increased glucosinolates and decreased antioxidant activity. In another study, the increasing amount of water in ethanol, acetone, and methanol extraction solvents extracted high amounts of flavonoids and phenolics. ${ }^{28}$ This showed how different factors could be a factor for the plant's phytochemical and biological activity.

The nature of the phytochemical constituents found in the extracts had exhibited akapulko's possible antifungal activity. Compounds such as the flavones 2,5,7,4'-tetrahydroxy isoflavone and 3,5,7,4'-tetrahydroxy flavone effectively inhibited T. schoenleinii, T. longiforus, Pseudallescheria boydii, C. albicans, and $A$. niger. ${ }^{30}$ In addition, anthraquinones such as aloe-emodin and emodin inhibited the growth of T. rubrum, T. mentagrophytes, and $M$. gypseum ${ }^{18,31,32}$ while cannabinoid alkaloid (4-butylamine 10-methyl6-hydroxy cannabinoid dronabinol) from ethanolic seed extract inhibited $C$. albicans and $A$. niger growth. ${ }^{33}$ Table 1 summarizes the phytochemicals detected from the different parts of akapulko using different types of solvents.

There has been very few published works on the mechanism of action of akapulko. Determining the molecular mechanism of action especially for crude herbal extracts is difficult because there are thousands of compounds present that can either act synergistically or antagonistically with each other. In three Cassia species (C. alata, C. fistula, and C. tora), the extracts inhibited hyphal growth in $T$. rubrum, $M$. gypseum, and P. marneffei, as well as the conidial growth in $M$. gypseum. The observed shrinking of the macroconidia and hyphae of extract-treated $M$. gypseum suggested cell leakage and change in membrane stability. ${ }^{48}$ Abnormality in the cell wall was also observed in methicillinresistant $S$. aureus cells ${ }^{34}$ upon treatment using the extract. In other plants, berberine (an alkaloid) destabilizes the cell wall and the synthesis of ergosterol and causes mitochondrial dysfunction in $C$. albicans. ${ }^{49}$ Alkaloids can also inhibit alpha-amylase in $A$. niger preventing the conversion of starch to usable forms of energy, impeding fungal growth. ${ }^{50}$ At a molecular level, in silico molecular docking, and in vitro HPLC-UV analyses showed that the compound astragalin (kaempferol-3-O- $\beta$-D-glucopyranosi de) binds to the DNA at the G-C base pairs. ${ }^{51}$ Moreover, in $S$. cerevisiae mutants lacking enzymes for antioxidant synthesis, DNA repair enzymes (RAD3, RADS2, and RAD6) or membrane constituents showed high sensitivity towards the akapulko extract. ${ }^{51}$ 
Table 1. Summary of the phytochemicals extracted from different plant parts of Senna alata Linn. using various solvent systems

\begin{tabular}{|c|c|c|}
\hline Chemical Compound & Plant Part & Extraction Solvent \\
\hline \multirow[t]{5}{*}{ Alkaloid } & Fresh and dried leaves & $96 \%$ ethanol ${ }^{34}$ \\
\hline & Leaf & $50 \%$ ethanol, $50 \%$ methanol, aqueous ${ }^{35}$ \\
\hline & Leaf, stem, root & Methanol, aqueous ${ }^{36}$ \\
\hline & Leaf & Ethanol ${ }^{37}$ \\
\hline & Leaf & Methanol, chloroform, petroleum ether, water ${ }^{38}$ \\
\hline Anthracenosides & Leaf & Methanol $^{39}$ \\
\hline Anthraquinone & Leaf & Methanol, chloroform, petroleum ether, water ${ }^{38}$ \\
\hline$\beta$-sitosterol & Leaf & Methanol $^{40}$ \\
\hline Cardioglycoside & Leaf, stem, root & Acetone ${ }^{36}$ \\
\hline Coumarin & Leaf & $50 \%$ ethanol, $50 \%$ methanol, aqueous ${ }^{35}$ \\
\hline \multirow[t]{6}{*}{ Flavonoids } & Fresh and dried leaves & $96 \%$ ethanol ${ }^{34}$ \\
\hline & Leaf & $50 \%$ ethanol, $50 \%$ methanol, aqueous ${ }^{35}$ \\
\hline & Leaf, stem, root & Methanol, acetone, aqueous ${ }^{36}$ \\
\hline & Leaf & Ethanol ${ }^{37}$ \\
\hline & Leaf & Methanol, chloroform, petroleum ether, water ${ }^{38}$ \\
\hline & Leaf & Water, benzene, chloroform, methanol, petroleum ether ${ }^{41}$ \\
\hline Gallic tannins & Leaf & Methanol $^{39}$ \\
\hline \multirow[t]{3}{*}{ Glycosides } & Leaf & $50 \%$ ethanol, $50 \%$ methanol, aqueous ${ }^{35}$ \\
\hline & Leaf, stem, root & Methanol, acetone, aqueous ${ }^{36}$ \\
\hline & Leaf & Methanol, chloroform, petroleum ether, water ${ }^{38}$ \\
\hline Gum & Leaf, stem, root & Aqueous $^{36}$ \\
\hline \multicolumn{3}{|l|}{ Lipids } \\
\hline Fat oil & Leaf, stem, root & Acetone, chloroform ${ }^{36}$ \\
\hline Linoleic acid & Leaf & Methanol ${ }^{40}$ \\
\hline Linolenic acid & Leaf & Methanol $^{40}$ \\
\hline Monosaturated fatty acids & Leaf & Methanol $^{40}$ \\
\hline Myriatic acid & Leaf & Methanol ${ }^{40}$ \\
\hline Oleic acid & Leaf & Methanol $^{40}$ \\
\hline Palmitic & Leaf & Methanol ${ }^{40}$ \\
\hline Polyunsaturated fatty acids & Leaf & Methanol $^{40}$ \\
\hline Saturated fatty acids & Leaf & Methanol $^{40}$ \\
\hline Stearic acid & Leaf & Methanol ${ }^{40}$ \\
\hline Mucilage & Leaf, stem, root & Aqueous $^{36}$ \\
\hline \multirow[t]{3}{*}{ Phenols } & Leaf & $50 \%$ ethanol, $50 \%$ methanol, aqueous ${ }^{35}$ \\
\hline & Leaf & Ethanol ${ }^{37}$ \\
\hline & Leaf & Water, benzene, chloroform, methanol, petroleum ether ${ }^{41}$ \\
\hline Phytosterol & Leaf, stem, root & Chloroform, acetone, methanol ${ }^{36}$ \\
\hline Proanthocyanidin & Leaf & Benzyl mercaptan ${ }^{42}$ \\
\hline \multirow[t]{2}{*}{ Quinone } & Dried and fresh leaves & $96 \%$ ethanol ${ }^{34}$ \\
\hline & Leaf, stem, root & Chloroform, acetone, methanol, water ${ }^{36}$ \\
\hline Resin & Leaf, stem, root & Chloroform, Acetone ${ }^{36}$ \\
\hline \multirow[t]{6}{*}{ Saponins } & Fresh and dried leaves & $96 \%$ ethanol ${ }^{34}$ \\
\hline & Leaf & $50 \%$ ethanol, aqueous ${ }^{35}$ \\
\hline & Leaf, stem, root & Aqueous $^{36}$ \\
\hline & Leaf & Ethanol ${ }^{37}$ \\
\hline & Leaf & Methanol, chloroform, petroleum ether, water ${ }^{38}$ \\
\hline & Leaf & Methanol $^{39}$ \\
\hline \multirow[t]{3}{*}{ Steroids } & Fresh and dried leaves & $96 \%$ ethanol ${ }^{34}$ \\
\hline & Leaf & $50 \%$ ethanol, $50 \%$ methanol, aqueous ${ }^{35}$ \\
\hline & Leaf & Ethanol ${ }^{37}$ \\
\hline
\end{tabular}




\begin{tabular}{|c|c|c|}
\hline \multirow[t]{5}{*}{ Tannins } & Fresh and dried leaves & $96 \%$ ethanol ${ }^{34}$ \\
\hline & Leaf & $50 \%$ ethanol, $50 \%$ methanol, aqueous ${ }^{35}$ \\
\hline & Leaf, stem, root & Methanol, acetone, aqueous ${ }^{36}$ \\
\hline & Leaf & Ethanol ${ }^{37}$ \\
\hline & Leaf & Methanol, chloroform, petroleum ether, water ${ }^{38}$ \\
\hline \multirow[t]{2}{*}{ Terpenoid } & Leaf & $50 \%$ ethanol, $50 \%$ methanol, aqueous ${ }^{36}$ \\
\hline & Leaf, stem, root & Methanol, acetone, aqueous ${ }^{37}$ \\
\hline Volatile oil & Leaf & Methanol, chloroform, petroleum ether, water ${ }^{38}$ \\
\hline \multicolumn{3}{|l|}{ Specific Compounds } \\
\hline 2,5,7,4'-tetrahydroxyisoflavone & Leaf & $80 \%$ ethanol ${ }^{10}$ \\
\hline 3,5,7,4'-tetrahydroxyflavone & Leaf & $80 \%$ ethanol ${ }^{30}$ \\
\hline Aloe-emodin & Leaf & $80 \%$ methanol $^{43}$ \\
\hline Cassiaindoline & Leaf $^{44}$ & \\
\hline Danthron & Leaf & Water ${ }^{45}$ \\
\hline Dihydroxycinnamic acid & Leaf & $10: 1$ acetone:ethanol ${ }^{46}$ \\
\hline \multirow[t]{3}{*}{ Kaempferol } & Leaf & $80 \%$ methanol $^{43}$ \\
\hline & Leaf & Water ${ }^{44}$ \\
\hline & Leaf, stem, bean & Methanol ${ }^{47}$ \\
\hline Kaempferol-3-O- $\beta$-glucopyranoside & Leaf & $80 \%$ methanol $^{43}$ \\
\hline Kaempferol-3-O-gentioside & Leaf & $80 \%$ methano $^{43}$ \\
\hline Kaempferol-O-glucoside & Leaf & Water $^{45}$ \\
\hline Luteolin & Leaf, stem, bean & Methanol $^{47}$ \\
\hline Quercetin-O-glucoside & Leaf & Water $^{45}$ \\
\hline Rhein & Leaf & Water $^{45}$ \\
\hline
\end{tabular}

\section{In vitro studies confirming the antifungal properties of Senna alata}

In vitro studies showed that akapulko had antibacterial, ${ }^{36,44,52,53,54}$ anti-inflammatory, ${ }^{55,56}$ anti-oxidant, ${ }^{37,40,57,58}$ hepatoprotective, ${ }^{59}$ cardioprotective, ${ }^{60}$ anti-tumor ${ }^{61,62,63}$ and anti-malarial ${ }^{64}$ properties. The most extensively studied property of akapulko was its antifungal ability. In the Philippines, ointments and soaps were formulated to target skin fungal diseases. ${ }^{14}$ Table 2 summarizes the majority of the in vitro antifungal testing done on akapulko against a wide range of fungal species. As shown in the table, the different plant parts of akapulko extracted using different types of solvents showed varying antifungal properties against common fungal pathogens. Specifically, there is a wide range in the activities observed using agar well diffusion and broth dilution method. Different studies also had varying results, which could probably be due to the differences in the environmental and laboratory conditions during the in vitro testing, type of soil used where the plant was cultivated, weather condition during the time of sampling, and even the species of fungi being tested. It can also be observed that isolating a specific compound and using it to kill the fungal species showed lower activity compared with the crude extract. This was true for the activity of leaf anthraquinone aglycone against E. flocossum (29-fold lower), M. gypseum (31-fold lower), T. metangrophytes (58-fold lower), and T. rubrum (99-fold lower). These results entail the presence of compounds present in the herbal crude extract that act synergistically with each other to achieve maximum pharmacologic effect. This is also suggestive of the benefit of using herbal crude extracts for medication over synthetically-produced, individual compounds.

In general, ethanol extracts showed better activity compared to methanol, hexane, and aqueous extracts among all fungal species. Good antifungal activity was generally seen for Aspergillus spp, Candida albicans, Cryptococcus neoformans, Epidermophyton flocossum, Microsporum spp., Trichophyton $s p p$, and Penicillium marneffei with MICs ranging from $3.5 \mathrm{mg} / \mathrm{mL}$ to $125 \mathrm{mg} / \mathrm{mL}$. Such concentrations were achievable for topical formulations, thus akapulko is suited to treat cutaneous fungal infections.

\section{Evidence of Efficacy and Safety in Humans}

In the Philippines, several clinical trials and formulations were done in hospitals and laboratories to determine the efficacy of akapulko in human fungal infections. A systematic review was recently performed involving seven randomized controlled trials (RCT) wherein patients were treated with formulations of akapulko then compared with other antifungal agents. ${ }^{75}$ There was a comparable mycologic cure between akapulko and $25 \%$ sodium thiosulfate in four RCTs. The two RCTs showed an efficacy similar to terbinafine and ketoconazole. Adverse effects were mild in all treatment groups. This systematic review is also featured in this issue of Acta Medica Philippina and thus the clinical trials will not be discussed in detail in this review. 
Table 2. Summary of the results of in vitro antifungal studies for Senna alata Linn.

\begin{tabular}{|c|c|c|c|}
\hline Fungi & Antifungal assay, outcome measured & Plant part - Extraction Solvent Used & Result \\
\hline \multirow[t]{7}{*}{ Aspergillus flavus } & AWD, ZOI & Leaf - Ethanol ${ }^{65}$ & $22.1 \pm 0.1 \mathrm{~mm}$ \\
\hline & AWD, ZOI & Leaf - Aqueous ${ }^{65}$ & $20.1 \pm 0.1 \mathrm{~mm}$ \\
\hline & AWD, ZOI & Leaf - Methanol 66 & $10-20 \mathrm{~mm}$ \\
\hline & AWD, ZOI & Leaf - Aqueous ${ }^{66}$ & $10-20 \mathrm{~mm}$ \\
\hline & $\mathrm{BM}, \mathrm{MIC}$ & Flower - Aqueous ${ }^{67}$ & $10 \mathrm{mg} / \mathrm{mL}$ \\
\hline & Extrapolated from AWD & Root - Methanol ${ }^{68}$ & $50 \mathrm{mg} / \mathrm{mL}$ \\
\hline & Extrapolated from AWD & Leaf - Methanol 68 & $50 \mathrm{mg} / \mathrm{mL}$ \\
\hline \multirow[t]{6}{*}{ Aspergillus niger } & AWD, ZOI & Leaf - Ethanol ${ }^{65}$ & $25.2 \pm 0.3 \mathrm{~mm}$ \\
\hline & AWD, ZOI & Leaf - Aqueous ${ }^{65}$ & $27.2 \pm 0.2 \mathrm{~mm}$ \\
\hline & BM, MIC & Root - Methanol 69 & $50 \mathrm{mg} / \mathrm{mL}$ \\
\hline & BM, MIC & Leaf - Methanol ${ }^{69}$ & $50 \mathrm{mg} / \mathrm{mL}$ \\
\hline & Extrapolated from AWD & Leaf - Ethanol ${ }^{68}$ & $3.5 \mathrm{mg} / \mathrm{mL}$ \\
\hline & Extrapolated from AWD & Leaf - Aqueous ${ }^{68}$ & $32.4 \mathrm{mg} / \mathrm{mL}$ \\
\hline Aspergillus parasiticus & $\mathrm{BM}, \mathrm{MIC}$ & Flower - Aqueous ${ }^{67}$ & $15 \mathrm{mg} / \mathrm{mL}$ \\
\hline \multirow{11}{*}{ Candida albicans } & AWD, ZOI & Leaf - Ethanol ${ }^{65}$ & $18.2 \pm 0.2 \mathrm{~mm}$ \\
\hline & AWD, ZOI & Leaf - Aqueous ${ }^{65}$ & $14.1 \pm 0.1 \mathrm{~mm}$ \\
\hline & $\mathrm{DD}, \mathrm{ZOI}$ at $20 \mathrm{mg} / \mathrm{mL}$ & Leaf - Methanol 66 & $10-20 \mathrm{~mm}$ \\
\hline & $\mathrm{DD}, \mathrm{ZOI}$ at $20 \mathrm{mg} / \mathrm{mL}$ & Leaf - Aqueous ${ }^{66}$ & $10-20 \mathrm{~mm}$ \\
\hline & Extrapolated from ZOI & Leaf - Ethanol ${ }^{68}$ & $5.6 \mathrm{mg} / \mathrm{mL}$ \\
\hline & Extrapolated from ZOI & Leaf - Aqueous ${ }^{68}$ & $26.9 \mathrm{mg} / \mathrm{mL}$ \\
\hline & BM, MIC & Leaf - Methanol ${ }^{69}$ & $35 \mathrm{mg} / \mathrm{mL}$ \\
\hline & BM, MIC & Root - Methanol 69 & $25 \mathrm{mg} / \mathrm{ml}$ \\
\hline & AWD, ZOI at $50 \mathrm{mg} / \mathrm{mL}$ & Leaf - Ethyl acetate ${ }^{70}$ & $12 \mathrm{~mm}$ \\
\hline & AWD, ZOI at $50 \mathrm{mg} / \mathrm{mL}$ & Leaf - Hexane ${ }^{70}$ & $15 \mathrm{~mm}$ \\
\hline & $\mathrm{BM}, \mathrm{MIC}$ & Flower - Aqueous ${ }^{67}$ & $15 \mathrm{mg} / \mathrm{mL}$ \\
\hline \multirow[t]{2}{*}{ Cryptoccocus neoformans } & BM, MIC & Root - Methanol 69 & $6 \mathrm{mg} / \mathrm{mL}$ \\
\hline & $\mathrm{BM}, \mathrm{MIC}$ & Leaf - Methanol ${ }^{69}$ & $13 \mathrm{mg} / \mathrm{mL}$ \\
\hline \multirow[t]{3}{*}{ Epidermophyton floccosum } & $\mathrm{BM}, \mathrm{MIC}$ & Stem bark - Ethanol ${ }^{71}$ & $2.5 \mathrm{mg} / \mathrm{mL}$ \\
\hline & BM, MIC & Leaf - Ethanol ${ }^{31}$ & $3.75 \mathrm{mg} / \mathrm{mL}$ \\
\hline & $\mathrm{BM}, \mathrm{MIC}$ & $\begin{array}{l}\text { Leaf - Antraquinone aglycone from } \\
\text { glycosidic fraction }{ }^{31}\end{array}$ & $0.13 \mathrm{mg} / \mathrm{mL}$ \\
\hline Microsporum canslaslomyces & BM, MIC & Stem bark - Ethanol ${ }^{71}$ & $2.5 \mathrm{mg} / \mathrm{mL}$ \\
\hline \multirow[t]{3}{*}{ Microsporum canis } & BM, MIC & Leaf - Ethanol ${ }^{72}$ & $62.5 \mathrm{mg} / \mathrm{mL}$ \\
\hline & Extrapolated from ZOI & Leaf - Ethanol ${ }^{68}$ & $12.6 \mathrm{mg} / \mathrm{mL}$ \\
\hline & Extrapolated from ZOI & Leaf - Aqueous ${ }^{68}$ & $30.30 \mathrm{mg} / \mathrm{mL}$ \\
\hline \multirow[t]{4}{*}{ Microsporum gypseum } & BM, MIC & Leaf - Ethanol ${ }^{72}$ & $62.5 \mathrm{mg} / \mathrm{mL}$ \\
\hline & BM, MIC & Leaf - Ethanol ${ }^{31}$ & $10.42 \mathrm{mg} / \mathrm{mL}$ \\
\hline & $\mathrm{BM}, \mathrm{MIC}$ & $\begin{array}{l}\text { Leaf - Anthraquinone aglycone from } \\
\text { glycosidic fraction }{ }^{31}\end{array}$ & $0.34 \mathrm{mg} / \mathrm{mL}$ \\
\hline & $100 \%$ hyphal growth inhibition, $I_{50}$ & Leaf - Methanol ${ }^{48}$ & $10 \mathrm{mg} / \mathrm{mL}, 0.8 \mathrm{mg} / \mathrm{mL}$ \\
\hline \multirow[t]{4}{*}{ Microsporum audouinii } & AWD, ZOI & Leaf - Hexane 22 & $25 \mathrm{~mm}$ \\
\hline & BM, MIC & Leaf -Ethyl Acetate ${ }^{22}$ & $22 \mathrm{~mm}$ \\
\hline & BM, MIC & Flower - Aqueous ${ }^{67}$ & $15 \mathrm{mg} / \mathrm{mL}$ \\
\hline & $\mathrm{BM}, \mathrm{MIC}$ & Leaf - Methanol ${ }^{73,74}$ & $25 \mathrm{mg} / \mathrm{mL}$ \\
\hline Trichophyton verrucosum, & $\mathrm{BM}, \mathrm{MIC}$ & Stem bark - Ethanol ${ }^{71}$ & $2.5 \mathrm{mg} / \mathrm{mL}$ \\
\hline Trichophyton megnini & BM, MIC & Leaf - Methanol 75 & $50 \mathrm{mg} / \mathrm{mL}$ \\
\hline
\end{tabular}




\begin{tabular}{|c|c|c|c|}
\hline \multirow[t]{11}{*}{ Trichophyton mentagrophytes } & BM, MIC & Stem bark - Ethanol ${ }^{71}$ & $2.5 \mathrm{mg} / \mathrm{mL}$ \\
\hline & $\mathrm{BM}, \mathrm{MIC}$ & Leaf - Ethanol ${ }^{31,72}$ & $125 \mathrm{mg} / \mathrm{mL}$ \\
\hline & BM, MIC & Leaf - Ethanol ${ }^{68}$ & $9.8 \mathrm{mg} / \mathrm{mL}$ \\
\hline & BM, MIC & Leaf - Aqueous ${ }^{68}$ & $27.8 \mathrm{mg} / \mathrm{mL}$ \\
\hline & Extrapolated from ZOI BM, MIC & Leaf - Ethanol ${ }^{31}$ & $19.64 \mathrm{mg} / \mathrm{mL}$ \\
\hline & Extrapolated from ZOI BM, MIC & $\begin{array}{l}\text { Leaf - Anthraquinone aglycone from } \\
\text { glycosidic fraction }{ }^{31}\end{array}$ & $0.34 \mathrm{mg} / \mathrm{mL}$ \\
\hline & BM, MIC & Leaf - Methanol ${ }^{74}$ & $14 \mathrm{~mm}$ \\
\hline & AWD, ZOI at $50 \mathrm{mg} / \mathrm{mL}$ & Leaf - Hexane $^{70}$ & $16 \mathrm{~mm}$ \\
\hline & AWD, ZOI at $50 \mathrm{mg} / \mathrm{mL}$ & Leaf - Ethyl acetate ${ }^{70}$ & $22 \mathrm{~mm}$ \\
\hline & $\mathrm{AWD}, \mathrm{ZOI}$ at $50 \mathrm{mg} / \mathrm{mL}$ & Leaf -Chloroform ${ }^{70}$ & $23 \mathrm{~mm}$ \\
\hline & AWD, ZOI & Leaf - Hexane 22 & $22 \mathrm{~mm}$ \\
\hline \multirow[t]{5}{*}{ Trichophyton rubrum } & BM, MIC & Leaf - Ethanol ${ }^{31}$ & $18.75 \mathrm{mg} / \mathrm{mL}$ \\
\hline & $\mathrm{BM}, \mathrm{MIC}$ & $\begin{array}{l}\text { Leaf -Anthraquinone aglycone from } \\
\text { glycosidic fraction }{ }^{31}\end{array}$ & $0.19 \mathrm{mg} / \mathrm{mL}$ \\
\hline & BM, MIC & Leaf - Methanol73 & $50 \mathrm{mg} / \mathrm{mL}$ \\
\hline & $\mathrm{DD}, \mathrm{ZOI} 20 \mathrm{mg} / \mathrm{mL}$ & Leaf - Methanol67 & $10-20 \mathrm{~mm}$ \\
\hline & $100 \%$ hyphal growth inhibition, $I C_{50}$ & Leaf - Methanol ${ }^{48}$ & $10 \mathrm{mg} / \mathrm{mL}, 0.5 \mathrm{mg} / \mathrm{mL}$ \\
\hline Trichophyton tonsurans & $\mathrm{BM}, \mathrm{MIC}$ & Leaf - Methanol ${ }^{73}$ & $50 \mathrm{mg} / \mathrm{mL}$ \\
\hline Penicillium marneffei & $100 \%$ hyphal growth inhibition, $I_{50}$ & Leaf - Methanol ${ }^{48}$ & $100 \mathrm{mg} / \mathrm{mL}, 6.6 \mathrm{mg} / \mathrm{mL}$ \\
\hline
\end{tabular}

AWD - agar well diffusion

ZOI - zone of inhibition

$D D$ - disc diffusion

$B M$ - broth microdilution

MIC - minimum inhibitory concentration

\section{CONCLUSION AND RECOMMENDATIONS}

Senna alata (akapulko) has been well-studied for its antifungal activity. Because of this, it is one of the ten herbal medicines endorsed by the Philippine Department of Health. Several in vitro and clinical studies confirmed its efficacy, thus, providing an evidence-based claim that can support and strengthen the use of this plant for the treatment of skin fungal indications. Still, additional studies on the formulation of the extract must be done, especially for these cases where fungal strains constantly change, phenotypically and genetically. Current formulations may not be effective in the coming years. Also, the cellular and molecular mechanisms of action of the plant extract must be established. Knowing the bioactive compounds can be of use to biotechnologists and breeders to produce plant varieties that can produce more of these compounds or can grow more of the plant parts collected for extraction that can contribute largely in conserving our resources. Lastly, the results of the molecular mechanism of action studies may give essential results in the formulation of personalized medications and treatment.

\section{Statement of Authorship}

All authors participated in data collection and analysis, and approved the final version submitted.

\section{Author Disclosure}

All authors declared no conflict of interest. The NIRPROMP-IHM has a utility model on an akapulco antifungal product.

\section{Funding Source}

This paper was funded by the authors.

\section{REFERENCES}

1. Brandt ME, Park BJ. Think fungus-prevention and control of fungal infections. Emerg Infect Dis. 2013;19(10):1688-9.

2. Fleming RV, Walsh TJ, Anaissie EJ. Emerging and less common fungal pathogens. Infect Dis Clin North Am. 2002; 16(4):915-33.

3. Handog EB, Dayrit JF. Mycology in the Philippines, revisited. Jpn J Med Mycol. 2005; 46(2):71-6.

4. Batac MCR, Denning D. Serious fungal infections in the Philippines. Eur J Clin Microbiol Infect Dis. 2017; 36(6):937-41.

5. Van Thiel DH, George M, Moore CM. Fungal infections: their diagnosis and treatment in transplant recipients. Int J Hepatol. 2012; 2012 (ID 106923):1-19.

6. Arif T, Bhosale JD, Kumar N, Mandal TK, Bendre RS, Lavekar GS, et al. Natural products - antifungal agents derived from plants. J Asian Nat Prod Res. 2009; 11(7):621-38.

7. Gallis HA, Drew RH, Pickard WW. Amphotericin B: 30 years of clinical experience. Rev Infect Dis. 1990; 12(2):308329.

8. El-Garhy OH. An overview of the azoles of interest. Int J Curr Pharm Res. 2015; 7(1):1-6.

9. Fanos V, Cataldi L. Amphotericin B-induced nephrotoxicity: a review. J Chemother. 2000; 12(6):463-70.

10. Sabra R, Branch RA. Amphotericin B nephrotoxicity. Drug Saf. 1990; 5(2):94-108. 
11. Venkatakrishnan K, Von Moltke LL, Greenblatt DJ. Effects of the antifungal agents on oxidative drug metabolism: clinical relevance. Clin Pharmacokinet. 2000; 38(2):111-80.

12. Sandhu SS, Shukla H, Aharwal RP, Kumar S, Shukla S. Antifungal azole derivatives and their pharmacological potential: prospects and retrospects. The Natural Products Journal. 2014; 4(2):1-13.

13. Adelowo F, Oladeji O. An overview of the phytochemical analysis of bioactive compounds in Senna alata. Advances in Biochemistry. 2017; 5(5): 102-9.

14. Maramba-Lazarte CC, Cortes-Maramba NP, Palacpac JB, et al. Guidebook on the proper use of medicinal plants. Manila: National Integrated Research Program on Medicinal Plants, Institute of Herbal Medicine, National Institutes of Health, University of the Philippines Manila; 2017. pp. 56-57.

15. Gritsanaphan W, Nualkaew S. Variation of anthraquinone content in Cassia surattensis. Warasan Phesatchasat. 2001; 28: 28-34.

16. Hennebelle T, Weniger B, Joseph H, Sahpaz S, Bailleul F. Senna alata. Fitoterapia. 2009; 80(7):385-93.

17. Giron LM, Freire V, Alonzo A, Caceres A. Ethnobotanical survey of the medicinal flora used by the Caribs of Guatemala. J Ethnopharmacol. 1991; 34(2-3):173-87.

18. Palanichamy S, Nagarajan S. Antifungal activity of Cassia alata leaf extract. J Ethnopharmacol. 1990; 29(3):337-40.

19. Parsons WT, Cuthberston EG. Noxious weeds of Australia. Melbourne: Indata Press; 1992.

20. Essiett UA, Bassey IE. Comparative phytochemical screening and nutritional potentials of the flowers (petals) of Senna alata (L.) Roxb., Senna hirsuta (L.) Irwin and Barneby, and Senna obtusifolia (L.) Irwin and Barneby (Fabaceae). J Appl Pharm Sci. 2013; 3(8):97-101.

21. Chatterjee S, Chatterjee S, Dutta S. An overview on the ethnophytopathological studies of Cassia alata - an important medicinal plant and the effect of VAM on its growth and productivity. International Journal of Research in Botany. 2012; 2(4):13-9.

22. Matthew OA, Gambo NN. Phytochemistry and antimicrobial activity of the leaf of Cassia alata Linn. Chemistry and Materials Research. 2013; 3(3):96-101.

23. Bhat RB, Eterjere EO, Oladipo VT. Ethnobotanical studies from central Nigeria. Econ Bot. 1990; 44(3):382-90.

24. Etukudo I. Ethnobotany: Conventional and Traditional Use of Plants. 1ed. Nigeria: Verdict Investment Ltd, Uyo; 2003. p.191.

25. Huang YC, Xiao D, Burton-Freeman M, Edirisinghe I. Chemical changes of bioactive phytochemicals during thermal processing. Reference module in Food Science. 2016.

26. Altemimi A, Lakhssassi N, Baharlouei A, Watson DG, Lightfoot DA. Phytochemicals: extraction, isolation, and identification of bioactive compounds from plant extracts. Plants (Basel). 2017; 6(4):E42.

27. Felhi S, Daoud A, Hajlaoui H, Mnafgui K, Gharsallah N, Kadri A. Solvent extraction effect on phytochemical constituents' profiles, antioxidant and antimicrobial activities and functional group analysis of Ecballium elaterium seeds and peels fruits. Food Sci Technol. 2017; 37(3):483-92.

28. Stalikas CD. Extraction, separation and detection methods for phenolic acids and flavonoids. J Sep Sci. 2007; 30(18):3268-95.

29. Scalzo RL, Picchi V, Migliori CA, Campanelli G, Leteo F, Ferrari $\mathrm{V}$, et al. Variations in the phytochemical contents and antioxidant capacity of organically and conventionally grown Italian cauliflower (Brassica oleracea L. subsp. Botrytis): Results from a three-year field study. J Agric Food Chem. 2013; 61(43):10335-44.

30. Rahman MS, Hasan AM, Ali MY, Ali MU. A flavone from the leaves of Cassia alata. Bangladesh J Sci Ind Res. 2008; 41(1-2):93-6.

31. Wuthi-udomlert M, Kupittayanant P, Gritsanapan W. In vitro evaluation of antifungal activity of anthraquinone derivatives of Senna alata. J Health Res. 2010; 24(3):117-22.

32. Sakunpak A, Sirikatitham A, Panichayupakaranant P. Preparation of anthraquinone high-yielding Senna alata extract and its stability. Pharm Biol. 2009; 47(3):236-41.

33. Okwu DE, Nnamdi FU. Cannabinoid dronabinol alkaloid with antimicrobial activity from Cassia alata Linn. Der Chemica Sinica. 2011; 2(2):247-54
34. Wikaningtyas P, Sukandar EY. The antibacterial activity study of Senna alata leaf extract and fraction towards MRSA (Methicillinresistant Staphylococcus aureus) and its mode of action. World J Pharm Pharm Sci. 2015; 4(4):126-33.

35. Meenupriya J, Sahaya Vinisha A, Priya P. Phytochemical screening and bioassay of Cassia alata leaf extract to study its skin hyperpigmentation activity. World J Pharm Sci. 2014; 2(12): 1723-7.

36. Sharma P, Pandey D, Rizvi AF, Gupta AK. Antimicrobial activity of Cassia alata from Raipus region against clinical and MTCC isolates. Int J Curr Microbiol App Sci. 2015; 4(1):330-9.

37. Sujatha J, Asokan S. Studies on the antioxidant activity of ethanol extract of Cassia alata using FT-IR, HPLC, and GC-MS analysis. Int J Adv Res Biol Sci. 2017; 4(12):112-9.

38. Idu M, Oronsaye FE, Igeleke CL, Omonigho SE, Omogbeme OE, Ayinde BA. Preliminary investigation on the phytochemistry and antimicrobial activity of Senna alata L., leaves. J Appl Sci. 2006; 6(11):2481-5

39. Senga Kitumbe P, Nkanga Isalomboto C, Ngoro Vero, Welo Unya J, Opota Onya D. Formulation of a herbal dermal ointment using whole leaves of Cassia alata Linn. Int J Ayurvedic Herb Med. 2016; 6(6):2355-63

40. Ali MI, Aboul-Enein AM, Mohamed SM, Abou Elella FM, Mohammed MMD, Hamed AR. Phytochemical, cytotoxicity and antioxidant investigation of Cassia alata leaves growing in Egypt. J Innov Pharm Biol Sci. 2017; 4(4): 97-105.

41. Pamulaparthi A, Prathap VR, Banala M, Nanna RS. Total phenolic, flavonoid contents and antioxidant assays in leaf extracts of Senna alata (L.) Roxb. J Pharm Sci \& Res. 2016; 8(9): 981-5.

42. Ramsay A, Mueller-Harvey I. Senna alata leaves are a good source of propelargonidins. Nat Prod Res. 2016; 30(13):1548-51.

43. Hazni H, Ahmad N, Hitotsuyanagi Y, Takeya K, Choo CY. Phytochemical constituents from Cassia alata with inhibition against Methicillin-Resistant Staphylococcus aureus (MRSA). Planta Med. 2008; 74(15):1802-5.

44. Villaseñor IM, Sanchez AC. Cassiaindoline, a new analgesic and anti-inflammatory alkaloid from Cassia alata. Z Naturforsch C J Biosci. 2009; 65(45-6):335-8.

45. Saito ST, Trentin Dda S, Macedo AJ, Pungartnik C, Gosmann G, Silveira Jde D, et al. Bioguided fractionation shows Cassia alata extract to inhibit Staphylococcus epidermidis and Pseudomonas aeruginosa growth and biofilm formation. Evid Based Complement Alternat Med. 2012; 2012:867103.

46. Paul B, Mitra P, Ghosh T, Salhan R, Singh TA, Chakrabatri A, et al. Isolation and structural determination of an antibacterial constituent from the leaves of Cassia alata Linn. J Pharmacogn Phytochem. 2013; 2(1):326-33.

47. Wahab A, Tahira, Begum S, Ayub A, Mahmood I, Mahmood T, et al. Luteolin and kaempferol from Cassia alata, antimicrobial and antioxidant activity of its methanolic extracts. FUUAST J Biol. 2014; 4(1):1-5.

48. Phogpaichit S, Pujenjob N, Rukachaisirikul V, Ongsakul M. Antifungal activity from leaf extracts of Cassia alata L., Cassia fistula L. and Cassia tora L. Songklanakarin J Sci Technol. 2004; 26(5):741-8.

49. Dhamgaye S, Devaux F, Vandeputte P, Khandelwal NK, Sanglard D, Mukhopadhyay G, et al. Molecular mechanisms of action of herbal antifungal alkaloid berberine in Candida albicans. PLoS One. 2014; 9(8):e104554.

50. Tintu I, Dileep KV, Augustine A, Sadasivan C. An isoquinoline alkaloid, berberine, can inhibit fungal alpha-amylase: enzyme kinetic and molecular modeling studies. Chem Biol Drug Des. 2012; 80(4):554-60.

51. Saito S, Silva G, Santos RX, Gosmann G, Pungartnik C, Brendel M. Astragalin from Cassia alata induced DNA adducts in vitro and repairable DNA damage in the yeast Saccharomyces cerevisiae. Int. J. Mol. Sci. 2012; 13(3):2846-62.

52. Paul B, Mitra P, Ghosh T, Salhan RN, Chakrabarti A, Singh TA, Das AP, et al. Effects of leaves of Cassia alata Linnaeus (Caesalpiniaceae) on the growth of Staphylococcus aureus Rosenbach. Pleione. 2012; 6(2):341-7 
53. Alalor CA, Igwilo CI, Jeroh E. Evaluation of the antibacterial properties of aqueous and methanol extracts of Cassia alata. Journal of Pharmacy and Allied Health Sciences. 2012; 2(2):40-6.

54. Valle Jr. DL, Andrade JI, Puzon JJM, Cabrera EC, Rivera WL. Antibacterial activities of ethanol extracts of Philippine medicinal plants against multidrug-resistant bacteria. Asian Pac J Trop Biomed. 2015; 5(7):532-40.

55. Lewis A, Levy A. Anti-inflammatory activities of Cassia alata extract in complete Freud's adjuvant arthritis in rats. West Indian Med J. 2011; 60(6): 615-21.

56. Da FL, Keugni AB, Belemtougri GR, Fotio TLA, Dimo T. Acute and subacute anti-inflammatory activities of dichloromethane extract of Cassia alata (Linn.) leaves in Wistar rats. Afr J Tradit Complement Altern Med. 2018; 15(1):174-82.

57. Sarkar B, Khodre S, Patel P, Mandaniya M. HPLC analysis and antioxidant potential of plant extract of Cassia alata. Asian Journal of Pharmaceutical Science and Technology. 2014; 4(1):4-7.

58. Panichayupakaranant S, Kaewsuwan S. Bioassay-guided isolation of the antioxidant constituent from Cassia alata L. leaves. Songklanakarin J Sci Technol. 2004; 26(1):103-7.

59. Anandan R, Jayakar B, Manavalan R. Hepatoprotective activity of the infusion of the dried leaves of Cassia alata Linn. Biomed Pharmacol J. 2009; 2(1):113-6.

60. Neharkar V, Jain DA, Pandhare R. Cardioprotective potential of Cassia alata (L.) leaves methanolic extract against Doxorubicin-induced cardiotoxicity in rats. IJPPR Human. 2016; 5(3):236-43.

61. Pieme CA, Penlap VN, Nkegoum B, Ngogang J. In vivo antioxidant and potential antitumor activity of aqueous ethanol extract of leaves of Senna alata (L.) Roxb (Caesalpiniaceae) on bearing carcinomatous cells. Int J Pharmacol. 2008; 4(4):245-51.

62. Pieme CA, Penlap VN, Ngogang J, Kuete V, Catros V, Moulinoux J Ph. In vitro effects of extract of Senna alata (Caesalpiniaceae) on the polyamines produced by Leukemia cells (L1210). Pharmacogn Mag. 2009; 5(17):8-13.

63. Olarte EI, Herrera AA, Villaseñor IM, Jacinto SD. In vitro antitumor properties of an isolate from leaves of Cassia alata L. Asian Pac J Cancer Prev. 2013; 14(5):3191-6.
64. Kayembe JS, Taba KM, Ntumba K, Tshiongo MTC, Kazadi TK. In vitro antimalarial activity of 20 quinones isolated from four plants used by traditional healers in the Democratic Republic of Congo. J Med Plants Res. 2010; 4(11):991-4.

65. Ogunjobi AA, Abiala MA. Antimicrobial activity of Senna alata and Phyllanthus amarus. Global Journal of Pharmacology. 2013; 7(2):198-202.

66. Makinde AA, Igoli JO, TA'Ama L, Shaibu SJ, Garba A. Antimicrobial activity of Cassia alata. Afr J Biotechnol. 2007; 6(13):1509-10.

67. Abubacker MN, Ramanathan R, Senthil Kumar T. In vitro antifungal activity of Cassia alata Linn. flower extract. Nat Prod Rad. 2008; 7(1):6-9.

68. Timothy SY, Wazis CH, Adati RG, Maspalma ID. Antifungal activity of aqueous and ethanolic leaf extracts of Cassia alata Linn. J Appl Pharm Sci. 2012; 2(7):182-5.

69. Ehiowemwenguan G, Inetianbor JE, Yakubu JM. Antimicrobial qualities of Senna alata. IOSR-JPBS. 2014; 9(2):47-52.

70. Villaseñor IM, Canlas AP, Pascua MP, Sabando MN, Soliven LA. Bioactivity studies on Cassia alata Linn. leaf extracts. Phytother Res. 2002; 16(Supp 1):S93-6.

71. Sule WF, Okonko IO, Omo-Ogun S, Nwanze JC, Ojezele MO, Ojezele OJ, et al. Phytochemical properties and in vitro antifungal activity of Senna alata Linn. crude stem bark extract. J Med Plant Res. 2011; 5(2):176-83.

72. Ibrahim D, Osman H. Antimicrobial activity of Cassia alata from Malaysia. J Ethnopharmacol. 1995; 45(3):151-6.

73. Ekwealor CC, Oyeka CA. In vitro anti-dermatophyte activities of crude extracts of Cassia alata. European Journal of Medicinal Plants. 2015; 5(3):255-9.

74. Aneke JN, Akalezi CO, Chibuike MI, Oguzie EE, Ukiwe LN. Proximate characterization of the methanolic extract of Cassia alata leaves. Afr J Plant Sci Biotechnol. 2010; 4(1):118-20.

75. Tababa EJL, Genuino RF, Gnilo CS. Senna alata (Akapulko) extract versus topical antifungals for treatment of superficial fungal skin infections: A systematic review and meta-analysis. Acta Med Philipp. 2020; 54(1):44-52.

\section{The Acta Medica Philippina is now accepting limited advertising for its front and back cover (colored), as well as for available spaces in some of its pages, as appropriate. For inquiries and submission of proposals, please email us at actamedicaphilippina.upm@up.edu.ph}

